\title{
Panel - Information Systems Qualitative Research in Health Care
}

\author{
B. Kaplan \\ Quinnipiac College, USA \\ F. Lau \\ University of Alberta, Canada
}

\section{J. Aarts}

Fontyns Hogeschölen, The Netherlands

\author{
D. E. Forsythe \\ University of California, San Francisco, USA
}

Interest in information systems in health care is growing. Information technology is becoming increasingly important as health care organizations feel pressures to improve quality while reducing costs. In addition to the more familiar business and administrative applications, information technology also is used to collect, analyze, and communicate clinical data in support of patient care processes. For example, there are systems for medical records, for communicating treatment orders or laboratory test results, for organizing and disseminating health knowledge as practical guidelines, for enhancing clinical practice through decision support systems, and for real-time monitoring of patient conditions.

An increasing number of information systems researchers throughout the world are carrying out studies in health care organizations. While the information systems field has developed, the discipline of medical informatics also has developed, a discipline that includes interest in organizational aspects as well as other areas.

The panel brings together information systems and medical informatics researchers experienced in different national health care settings. Panelists will draw on a variety of research projects to provide empirically based discussion of why qualitative 
research is important in health care and how to derive more general lessons for qualitative information systems research in other substantive areas. Panelists will draw on their research experiences to discuss

- the appropriateness of qualitative research in health care;

- issues unique to the intersection of health care and information systems qualitative research; and

- general issues of information systems qualitative research as they are exemplified in health care settings.

Bonnie Kaplan will chair and moderate the panel. She will make introductory remarks that set the panel agenda and closing remarks that tie together panelists' comments and provide a framework for opportunities and experiences in qualitative research in health care. She will raise issues in conducting information systems qualitative research in hospitals, such as legitimacy of the researcher, gaining entry, and presentation of results. She will draw upon research findings from a variety of hospital-based research projects she conducted to suggest topics and theoretical frameworks relevant to the IS research community at large.

Francis Lau will present an example of qualitative IS research in health care. He will discuss a project in which he and other researchers are studying the adoption and use of an Internet-based disease guidance system by physicians, residents, and nurses. The project provides an example of how IS qualitative research can be applied in health care by illustrating the development and refinement of such qualitative methodologies as action research, ethnography, longitudinal research, and phenomenolgy.

Jos Aarts will present a theoretical basis for qualitative research projects in information systems using health care settings as an example. He will discuss the social nature of clinical work. He will present a conceptual model that relates organizational change and the planning, design, and implementation of information systems to clinical work. Based on that, he will present the types of qualitative methodologies most appropriate for assessing the impact of information and communication technology in health care delivery. He will also propose an agenda for IS research in health care settings. The models and methodologies he suggests should be of general interest to the IS community because of the social nature of work in other settings.

Diana Forsythe will discuss what constitutes ethnographic expertise and why it is useful in system design and evaluation based on her nine years of research in software development laboratories where well-known medical informatics developers turned ethnographers. Forsythe's characterization of the misconceptions involved in such do-it-yourself ethnography will be recognizable to IS researchers working in other contextual settings. Her discussion of some do's and don'ts of ethnographic research will shed light on the legitimacy of qualitative research in IS. 